\title{
Effects of Feeding Probiotics on the Localization of Cells Containing Immunoreactive Interleukin-6 in the Intestine of Broiler Chicks
}

\author{
Yukinori Yoshimura, Miwa Oda and Naoki Isobe \\ Graduate School of Biosphere Science, Hiroshima University, Higashi-Hiroshima 739-8528, Japan
}

\begin{abstract}
The aim of this study was to examine whether treatments of chicks with probiotics affect the population of cells containing interleukin-6 (IL-6) and IgA in the intestine of broiler chicks. The diets with probiotics, Streptococcus faecalis, Clostridium buthricum, Bacillus mesentericus, were fed from day 0 to 10 of age. The sections of duodenum, pouch and tonsil region of cecum and colon were immunostained for IL-6 and IgA. The IL-6 immunoreaction products were identified in the leukocytes locating in the subepithelial tissues under the mucosal and crypt epithelium and lamina propria, and occationally in the the mucosal and crypt epithelium, in all birds. The density of immunoreactive (ir) IL-6 cell population in the mucosa tended to be lowest in the duodenum and highest in the colon. The density did not differ between control and probiotics groups on day 5; however, that in the cecum pouch and colon was significantly greater in the probiotics group than control on day 10. The IgA immunoreaction products were identified in the mucosal and crypt epithelial cells in all segments of the intestine, and some IgA-positive cells were localized in the lymphoid tissue in the cecum tonsil, whereas differences in their localization were not observed between probiotics and control groups. In conclusion, we suggest that probiotics increases the population of irIL- 6 cells in the cecum and colon mucosal tissues of chicks. Because IL-6 is multifunctional cytokine for immune system, the increased IL-6 secretion may affect the immune functions in the intestinal mucosal tissues.
\end{abstract}

Key words: chicks, immunoglobulin A, interleukin-6, intestine, probiotics

J. Poult. Sci., 47: 250-255, 2010

\section{Introduction}

Gastrointestinal immune system plays essential role to protect the tissues from infection by pathogenic microorganisms in feed. Mucosal immunity mediated by $\mathrm{T}$ and $\mathrm{B}$ cells is well developed in the intestine, whereas IgA secreted from the mucosa is one of the most important molecules for the defense system (Brandtzaeg, 2009). Although interleukin-6 (IL-6) is a proinflammatory cytokine, it also shows multifunctional natures including regulation of the immune response (Schneider et al., 2001; Sevimli et al., 2008). It is reported in mammals that IL-6 stimulates $\mathrm{CD}^{+}{ }^{+} \mathrm{Th} 2$ differentiation and inhibit Th1 differentiation (Diehl et al., 2000), and also it switches the differentiation of monocytes from dendritic cells to macrophages (Chomarat et al., 2000). The IL-6 also plays a critical role in vivo in the development of local IgA antibody responses (Ramsay et al., 1994).

Probiotics have the potential to impact health by affecting the colonization of microorganisms or interactivity with host cells in the gut (Lenoir-Wijnkoop et al., 2007;

Received: March 5, 2010, Accepted: May 12, 2010

Released Online Advance Publication: May 25, 2010

Correspondence: Yukinori Yoshimura, Graduate School of Biosphere Science, Hiroshima University, Higashi-Hiroshima 739-8528, Japan. (E-mail: yyosimu@hiroshima-u.ac.jp)
Mountzouris et al., 2009; Taheri et al., 2009). In unimmunized chicks, probiotics enhanced the induction of natural antibodies in serum and intestine (Haghighi et al., 2006). Some probiotics from the Lactobacillus strains, such as Lactobacillus salivarius (L. salivarius) and $L$. plantarum combined with xylanase, reduced the number of pathogenic bacteria in the intestine (Vandeplas et al., 2009; Kizerwetter-Swida and Binek, 2009). Sato et al. (2009) reported that when the chicks were fed with immunobiotics, L. jensenii TL2937 or L. gasseri TL2919, T cell related mRNAs (CD-3, IL-2 and interferon- $\gamma$ ) and toll-like receptor (TLR) mRNAs were increased in the foregut. However, it remains to be investigated whether probiotics affect the IL- 6 producing cells that exert multifunctions including the regulation of $\operatorname{IgA}$ synthesis and other immune responses.

Thus, the aim of this study was to examine whether treatments of chicks with probiotics affects the population of IL-6 containing cells in the intestine. The effects of probiotics feeding on the IgA localization that may be affected by IL- 6 were also examined. Because the cecum is one of the intestinal segments where many of the microorganisms colonize, the analysis was performed in the cecum tissue in addition to duodenum and colon. 


\section{Materials and Methods}

\section{Treatment of Birds and Tissue Collection}

Day-old male broiler chicks (Chunky broilers) were purchased from a local hatchery (Fukuda Poultry, Okayama, Japan). Birds were divided into two groups ( $n$ $=12$ each), namely control group (given only commercial starter diet; Nihon Nosan Kogyo Co. Ltd., Yokohama, Japan) and probiotics group (given commercial starter diet with probiotics). Probiotics used in this study was Toaraze for chickens (Toa Pharmaceutical Co., Ltd., Tokyo) that contained Streptococcus faecalis ( $>1 \times 10^{8}$ / g), Clostridium buthricum ( $\left.>1 \times 10^{7} / \mathrm{g}\right)$, Bacillus mesentericus $\left(>1 \times 10^{7} / \mathrm{g}\right)$, and it was added to the feed at a concentration of $0.2 \%(\mathrm{w} / \mathrm{w})$. They were maintained in a room with 24-h lighting and at a temperature of $30^{\circ} \mathrm{C}$. The birds were reared under ad libitum access to feed and water. On day 0 (before feeding, $n=6$ ), 5 and 10 , the intestines of both control and probiotics groups $(n=6$ for each group and on each day) were collected after euthanization of birds with carbon dioxide. Tissues were fixed with $10 \%(\mathrm{v} / \mathrm{v})$ formalin in phosphate buffered saline (PBS). Handling of chicks was done in accordance with the regulations of Hiroshima University for animal experiments.

\section{Tissue Preparation and Immunostaining}

The duodenum, pouch and tonsil regions of caecum, and colon fixed in $10 \%(\mathrm{v} / \mathrm{v})$ formalin were processed for paraffin sections. The sections ( $4 \mu \mathrm{m}$ thicknesses) were air-dried on MAS coated pre-cleaned slides (Matsunami Glass Inc. Osaka, Japan) and deparaffinized before staining.

For IL-6 immunostaining, the sections were autoclaved in $0.01 \mathrm{M}$ citric acid ( $\mathrm{pH}$ 6.0) for $1 \mathrm{~min}$ to activate antigen and then washed with PBS. The immunohistochemistry was performed using rabbit anti-chicken IL-6 polyclonal antibody (Abcam Co., Tokyo, Japan) and Vecta Stain ABC kit (Vector Lab., Burlingame, CA, USA). The slides were incubated with blocking solution $[1.5 \%$ (vol/ vol) normal goat serum in PBS] for $30 \mathrm{~min}$ at room temperature. Sections were incubated overnight with antibodies to IL-6 diluted at a concentration of 1: 100 in blocking solution, followed by washing with PBS $(3 \times 5$ $\mathrm{min})$. The sections were then incubated with biotinylated anti-rabbit IgG and avidin-biotin-peroxidase complex for $1 \mathrm{~h}$ each, and were washed with PBS $(3 \times 5 \mathrm{~min})$ after each step. Immunoprecipitates were visualized by incubating the sections with $\mathrm{DAB}-\mathrm{H}_{2} \mathrm{O}_{2}$ mixture $[0.02 \%$ (wt/vol) $3^{\prime}, 3^{\prime}$-diaminobenzidine tetrahydrochloride and $0.005 \%$ (vol/vol) $\mathrm{H}_{2} \mathrm{O}_{2}$ in $0.05 \mathrm{M}$ Tris- $\mathrm{HCl}$ ( $\mathrm{pH}$ 7.6)].

For IgA immunostaining, deparaffinizaed sections were incubated with $1 \%$ blocking reagent in PBS (Roche Diagnostics $\mathrm{GmbH}$, Mannheim, Germany) for $30 \mathrm{~min}$, and then overnight with goat anti-chicken $\operatorname{IgA}$ antibody (Bethyl Lab., Montgomery, TX, USA) diluted at a concentration of 1: 100 in 1\% blocking reagent. After washing in PBS, sections were incubated with biotinylated anti-goat IgG (Chemicon International, Inc., Temecula, CA, USA) and avidin-biotin-peroxidase complex in Vecta Stain ABC kit (Vector Lab.) for $1 \mathrm{~h}$ each. Then immunoprecipitates were visualized as described above using DAB- $\mathrm{H}_{2} \mathrm{O}_{2}$ mixture.

After immunostaining, the sections were counterstained with hematoxylin, and dehydrated with graded series of alcohols and mounted. Control staining was carried out simultaneously in which the first antibody was replaced with normal rabbit IgG or normal goat serum. Some sections were also stained with haematoxylin and eosin for the observation of the general tissue structure.

\section{Image Analysis}

The sections were examined under a light microscope (Nikon Eclipse E, Tokyo, Japan) using a Nomarsky filter. The populations of cells immunopositive for IL-6 (irIL-6 cell) in the mucosal tissue (lamina propria and surface epithelium) were analyzed under a light microscope with a computer assisted image analysis system (Image-Pro Plus; Media Cybernetics, Silver Spring, MD, USA). The irIL6 cells were counted in 2 different regions on one section, and the average number of cells in a $1 \times 10^{6} \mu \mathrm{m}^{2}$ area was then calculated. The IgA-positive cells were found in the epithelium. Because direction of tissue section affected the area values of epithelium, it was considered inadequate to analyze the cell number per unit area of the epithelium. Thus, we just evaluated the localization profiles of them by general observation under a microscope.

\section{Statistical Analysis}

Differences in the density of irIL-6 cells in the mucosal tissues and body weights between control and probiotics groups were examined by t-test, and those among the different intestinal segments within control or probiotics group were analyzed by Tukey Kramer test. Differences were considered significant when $\mathrm{P}$ value was $<0.05$.

\section{Results}

The body weights did not show significant difference between control and probiotics groups until on day 10 (Fig. 1). There were no histological abnormalities or inflammatory signs in the intestinal tissues of both groups

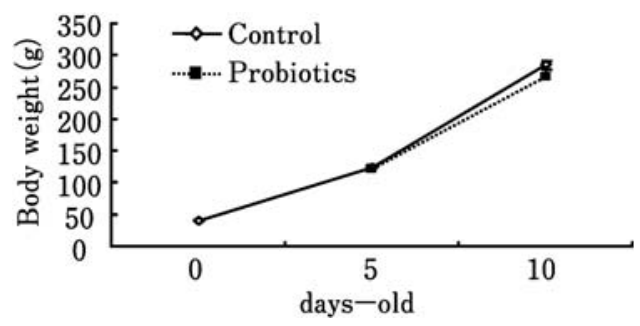

Fig. 1. Changes in the body weights of broiler chicks fed with or without probiotics from day 0 to 10 . Probiotics (Toaraze for chickens containing Streptococcus faecalis, Clostridium buthricum, Bacillus mesentericus) was mixed in commercial starter diet at a concentration of $0.2 \%$. Values are mean $\pm \mathrm{SE}(n=6)$. 
until day 10 (Data not shown).

The IL-6 immunoreaction products were identified in the leukocytes in the mucosal tissues of the intestine. Those irIL-6 cells were localized in the subepithelial tissues beneath the mucosal and crypt epithelium, and lamina propria in the duedenum, cecum pouch and tonsil, and colon in the birds of control and probiotics groups of day 10 (Fig. 2), and all birds on day 5 and day 0 (data not shown). Some irIL-6 cells were occasionally observed in the mucosal epithelium. The density of irIL- 6 cell population in the mucosa tended to be lowest in the duodenum and highest in the colon in both control and probiotics groups of day 0 to 10 (Fig. 3). The density did not differ between control and probiotics groups on day 5 ; however, that in the cecum pouch and colon was significantly greater in the probiotics group than control on day 10 (Fig. 3c).

Figure 4 shows the localization of immunoreaction products for IgA in the intestine of control and probiotics groups on day 10. In the control group, the immunoreaction products were identified in the mucosal and crypt epithelial cells in all the segments of intestine, whereas their density was greater in the duodenum (Fig. 4a) than other segments (Fig. 4c, e and f). Some IgA-positive cells were localized in the lymphoid tissue in the lamina propria of cecum tonsil (Fig. e), although they were only a few in the cecum pouch (Fig. 4c) and negligible in other segments (Fig. 4a and g). Same localization profiles of IgA immunoreaction products were also observed in the pro-

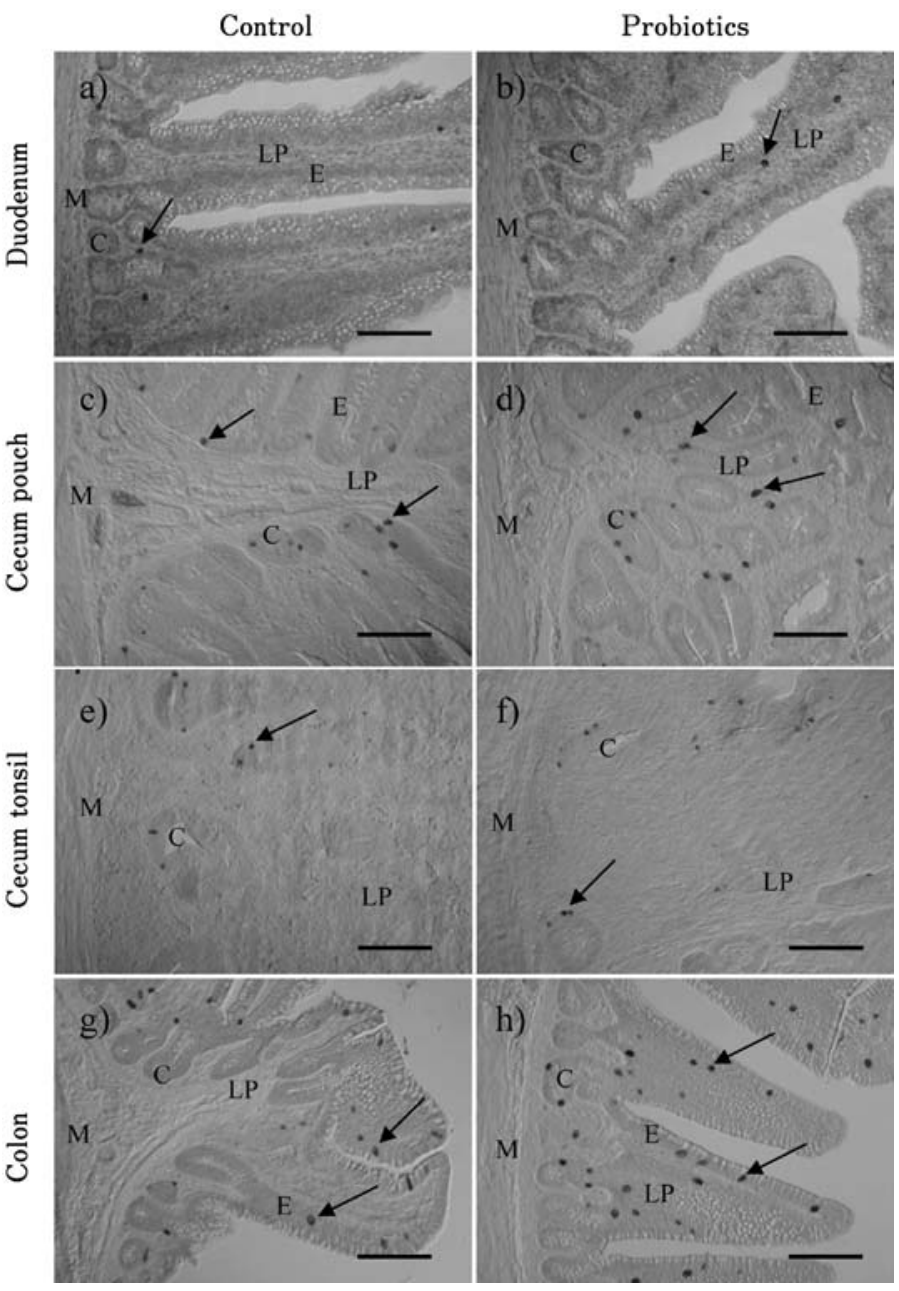

Fig. 2. Sections of intestinal tissues of 10-day-old chicks fed with control (a, c, e and g) or probiotics (b, d, f and h) diets immunostained for interleukin-6 (IL-6). a) and b), duodenum; c) and d), cecum pouch; e) and f), cecum tonsil; g) and h), colon. Note that IL-6 immunoreaction products are localized in the leukocytes distributing in the subepithelial tissues and lamina propria and occasionally in the mucosal epithelium (arrows). $\mathrm{C}=$ crypt, $\mathrm{E}=\mathrm{mu}$ cosal epithelium, $\mathrm{LP}=$ lamina propria, $\mathrm{M}=$ smooth muscle layer. Scale bars $=100 \mu \mathrm{m}$. 


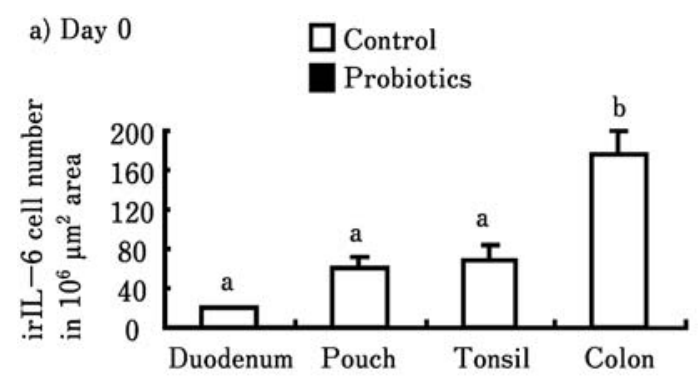

b) Day 5
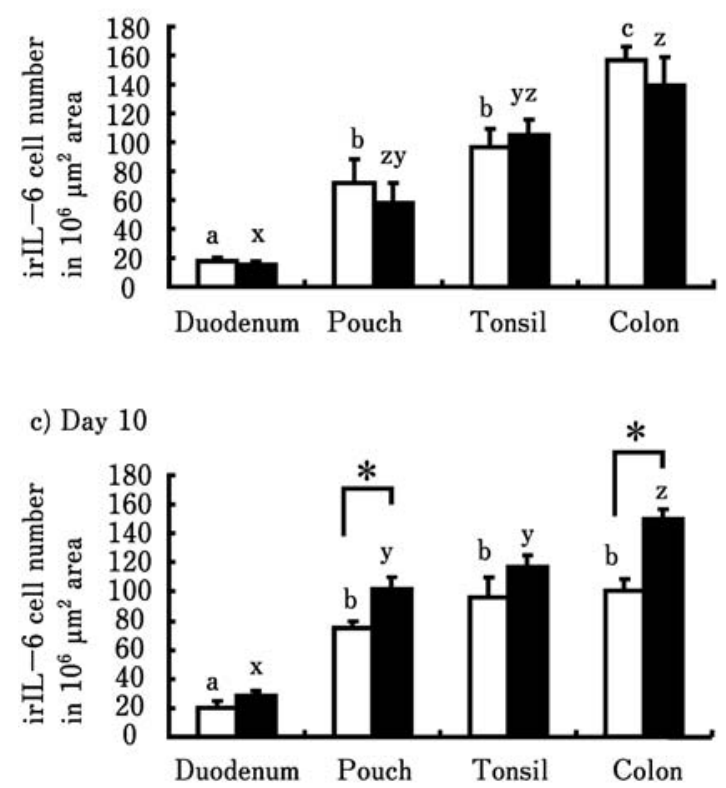

Fig. 3. Frequencies of cells containing immunoreactive interleukin-6 (IL-6) in the intestines of chicks fed with or without probiotics from day 0 to 10 . a), day 0 bird (before treatment with probiotics); b), day 5 bird; c), day 10 bird. Values are mean \pm SE $(n=6)$. a-c and $x-y$ : Values with different letters are significantly different within control or probiotics groups, respectively $(P<$ $0.05)$. *Values are significantly different between control and probiotics groups $(P<0.05)$.

biotics group (Fig. 4b, d, f and h). The IgA immunoreaction products were also identified in the mucosal and crypt epithelium in the birds of day 0 and 5 (data not shown).

\section{Discussion}

We have examined the effects of probiotics containing Streptococcus faecalis, Clostridium buthricum and Bacillus mesentericus on the population of irIL-6 cells and localization of $\operatorname{IgA}$ in the intestine of broiler chicks. Significant finding was that feeding with probiotics increased the density of irIL-6 cells in the cecum pouch and colon by day 10. No significant difference was observed in the body weight gain between control and probiotics group, and histological abnormalities or inflammatory signs were not observed in the intestinal tissues until day 10 . These results suggest that the probiotics used in this study caused no inflammation in the intestinal tissues.

The immunoreaction products of IL-6 were identified in the leukocytes, and those irIL-6 cells were located mainly in the subepithelial tissues beneath the mucosal and crypt epithelium and lamina propria. The density of IL-6 cells in the intestine was greater in the colon and cecum than duodenum in both control and probiotics groups. Thus, it is suggested that IL-6 mediated physiological events could occur in the intestinal mucosa, and more amount of IL-6 may be secreted in the lower intestine including cecum and colon than upper intestinal segments.

In the chicks fed with probiotics, mRNA expression of T cell-related molecules and TLRs in the gut, but not of B cell molecule, was increased (Sato et al., 2009). The current study also showed the increase of irIL- 6 cell density by probiotics in the cecum and colon. Thus, probiotics is likely to affect not only the intestinal bacteria balance (Lenoir-Wijnkoop et al., 2007) but also intestinal immune function in chicks. The increased irIL-6 cell population may results in the secretion of more amount of IL-6 in the chicks fed with probiotics. Although IL-6 is one of the proinflammatory cytokines (Schneider et al., 2001), increase of the irIL-6 cells in the intestine of chicks fed with probiotics may not induce inflammation or disorder of the tissue because no such histological signs were observed under histology.

It is reported that IL-6 stimulates the development of local IgA antibody response (Ramsay et al., 1994), IL-1 $\beta$ secretion (Staeheli et al., 2001), and Th2 cell differentiation (Davis et al., 1978; Diehl et al., 2000). The IgA is accepted to play a significant role in the mucosal immunity (Brandtzaeg, 2009). This class of immunoglobulin, which is synthesized by the plasma cells, binds to polymeric receptors expressed by mucosal epithelial cells and then secreted with the mucosal substances (Parry and Porter, 1978; Brandtzaeg, 2009). Haghighi et al. (2006) reported that when 1-day-old chicks were treated with probiotics, serum and intestinal antibodies in addition to intestinal IgA were increased in unimmunized 14-d-old chicks, suggesting that the treatment enhanced natural antibody production. The current study revealed that the immunoreaction products of $\operatorname{IgA}$ was present in the mucosal and crypt epithelium of day 0,5 and 10 chicks, whereas the IgA-positive plasma cells were seldom observed in the mucosal tissue other than lymphoid tissue in the cemum of day 10 chicks. Although the origin of IgA identified in the epithelium remains unknown, namely maternal or neonatal $\operatorname{IgA}$, the IgA may play significant role in mucosal immunity. In the current study, differences were not observed in the localization profiles of $\operatorname{IgA}$ immunoreaction products in the epithelial cells and IgA-positive plasma cells in the lymphoid tissue of the cecum between the control and probiotics groups until day 10 . Since natural antibody production including IgA was enhanced by the probiotics in 14-d-old chicks (Haghighi et al., 2006), further studies are necessary to examine whether 


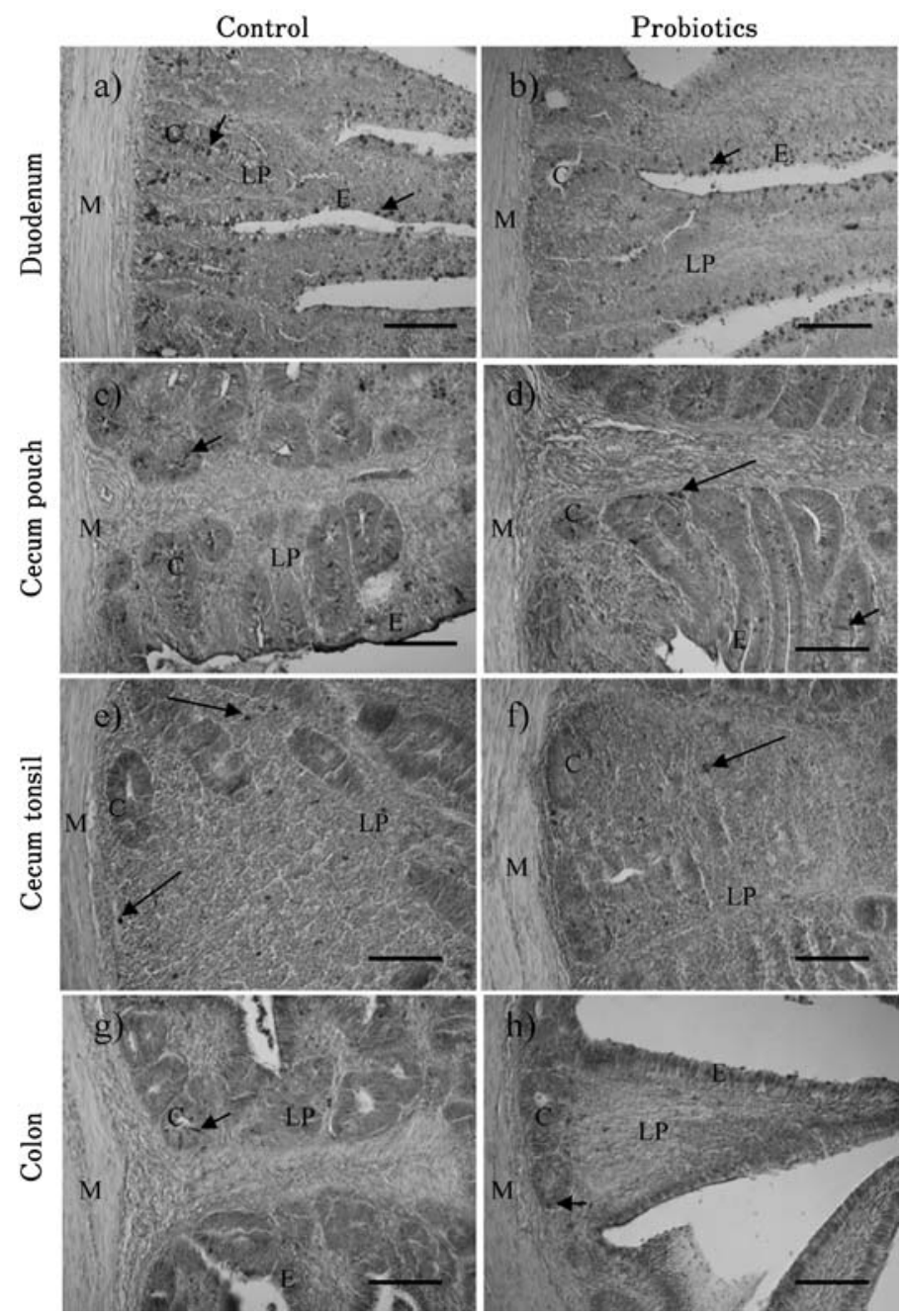

Fig. 4. Sections of intestinal tissues of 10-day-old chicks fed with control (a, c, e and g) or probiotics (b, d, f and h) diets immunostained for immunoglobulin A (IgA). a) and b), duodenum; c) and d), cecum pouch; e) and f), cecum tonsil; g) and h), colon. Note that IgA immunoreaction products are localized in the mucosal and crypt epithelium (short arrows). Long arrows show the IgA-positive plasma cells in the lymphoid tissues of cecum tonsil (e and f) and in the cecum lamina propria (d). See Fig. 2 for explanation of letterings on the micrographs. Scale bars $=100$ rem.

the effects of probiotics on the density of IgA molecules and IgA-positive cells appear in the more aged chicks in association with higher frequencies of IL-6 cells.

Probiotics increased the population of $\mathrm{CD}^{+}$and $\mathrm{CD} 8^{+}$ $\mathrm{T}$ cells followed by $\mathrm{CD} 4^{+} \mathrm{T}$ cells in the chick intestine (Noujaim et al., 2008), and expression of $\mathrm{T}$ cell related molecules (Sato et al., 2009). Although we have not examined the $\mathrm{T}$ cell functions, it is necessary to investigate whether the increased IL-6 secretion in the intestine of chicks fed with probiotics up-regulates the $\mathrm{T}$ cell population and functions in the intestine.

In conclusion, we suggest that probiotics increased the population of irIL- 6 cells in the cecum and colon mucosal tissues of chicks. Because IL-6 is a multifunctional cytokine for immune system, the increased IL-6 secretion may affect the immune functions in the intestinal mucosal tissues. Although differences in the localization of $\operatorname{IgA}$ immunoreaction products were not observed between probiotics and control groups until 10 days, effects of probiotics treatment for a longer term on their localization may be necessary to be studied.

\section{Acknowledgments}

We thank Dr. Takashi Bungo, Hiroshima University, 
for his valuable advice for this study. This work was financially supported by Kieikai Foundation Tokyo, Japan.

\section{References}

Brandtzaeg P. Mucosal immunity: induction, dissemination, and effector functions. Scandinavian Journal of Immunology, 70: 505-515. 2009.

Chomarat P, Banchereau J, Davoust J and Palucka AK. IL-6 switches the differentiation of monocytes from dendritic cells to macrophages. Nature Immunology, 1: 510-514. 2000.

Davis PJ, Parry SH and Porter P. The role of secretory IgA in anti-coccidial immunity in the chicken. Immunology, 34: 879-888. 1978.

Diehl S, Anguita J, Hoffmeyer A, Zapton T, Ihle JN, Fikrig E and Rincón M. Inhibition of Th1 differentiation by IL-6 is mediated by SOCS1. Immunity, 13: 805-815. 2000.

Haghighi HR, Gong J, Gyles CL, Hayes MA, Zhou H, Sanei B, Chambers JR and Sharif S. Probiotics stimulate production of natural antibodies in chickens. Clinical and Vaccine Immunology, 13: 975-980. 2006.

Kizerwetter-Swida M and Binek M. Protective effect of potentially probiotic Lactobacillus strain on infection with pathogenic bacteria in chickens. Polish Journal of Veterinary Sciences, 12: 15-20. 2009.

Lenoir-Wijnkoop I, Sanders ME, Cabana MD, Caglar E, Corthier G, Rayes N, Sherman PM, Timmerman HM, Vaneechoutte M, Van Loo J and Wolvers DA. Probiotic and prebiotic influence beyond the intestinal tract. Nutrition Reviews, 65: 469-489. 2007.

Mountzouris KC, Balaskas C, Xanthakos I, Tzivinikou A and Fegeros K. Effects of a multi-species probiotic on biomarkers of competitive exclusion efficacy in broilers challenged with Salmonella enteritidis. British Poultry Science, 50: 467-478. 2009.

Noujaim JC, Andreatti Filho RL, Lima ET, Okamoto AS, Amorim RL and Neto RT. Detection of T lymphocytes in intestine of broiler chicks treated with Lactobacillus spp. and challenged with Salmonella enterica serovar Enteritidis. Poultry Science, 87: 927-933. 2008.

Parry SH and Porter P. Characterization and localization of secretory component in the chicken. Immunology, 34: 471478. 1978.

Ramsay AJ, Husband AJ, Ramshaw IA, Bao S, Matthaei KI, Koehler $G$ and Kopf $M$. The role of interleukin-6 in mucosal IgA antibody responses in vivo. Science, 264: 561563. 1994.

Sato K, Takahashi K, Tohno M, Miura Y, Kamada T, Ikegami $\mathrm{S}$ and Kitazawa $\mathrm{H}$. Immunomodulation in gut-associated lymphoid tissue of neonatal chicks by immunobiotic diets. Poultry Science, 88: 2532-2538. 2009.

Schneider K, Klaas R, Kaspers B and Staeheli P. Chicken interleukin-6. cDNA structure and biological properties. European Journal of Biochemistry, 268: 4200-4206. 2001.

Sevimli A, Misirlioğlu D, Yağci A, Bülbül A, Yilmaztepe A and Altunbas K. The role of chicken IL- $1 \beta$, IL- 6 and TNF- $\alpha$ in the occurrence of amyloid arthropathy. Veterinary Research Communications, 32: 499-508. 2008.

Staeheli P, Puehler F, Schneider K, Göbel TW and Kaspers B. Cytokines of birds: conserved functions--a largely different look. Journal of Interferon and Cytokine Research, 21: 9931010. 2001.

Taheri HR, Moravej H, Tabandeh F, Zaghari M and Shivazad M. Screening of lactic acid bacteria toward their selection as a source of chicken probiotic. Poultry Science, 88: 15861593. 2009.

Vandeplas S, Dauphin RD, Thiry C, Beckers Y, Welling GW, Thonart $\mathbf{P}$ and Théwis A. Efficiency of a Lactobacillus plantarumxylanase combination on growth performances, microflora populations, and nutrient digestibilities of broilers infected with Salmonella Typhimurium. Poultry Science, 88: 1643-1654. 2009. 